\title{
DETECTING AND LOCATING PLAGIARISM OF MUSIC MELODIES BY PATH EXPLORATION OVER A BINARY MASK
}

\author{
Mu-Syuan Sie, Cheng-Chin Chiang, Hsiu-Chun Yang and Yi-Le Liu \\ Department of ComputerScience and Information Engineering \\ National Dong Hwa University, Taiwan
}

\begin{abstract}
To the best of our knowledge, the issues of automatic detection of music plagiarism have never been addressed before. This paper presents the design of an Automatic Music Melody Plagiarism Detection (AMMPD) method to detect and locate the possible plagiarism in music melodies. The key contribution of the work is an algorithm proposed to address the challenging issues encountered in the AMMPD problem, including (1) the inexact matching of noisy and inaccurate pitches of music audio and (2) the fast detection and positioning of similar subsegments between suspicious music audio. The major novelty of the proposed method is that we address the above two issues in temporal domain by means of a novel path finding approach on a binarized 2-D bit mask in spatial domain. In fact, the proposed AMMPD method can not only identify the similar pieces inside two suspicious music melodies, but also retrieve music audio of similar melodies from a music database given a humming or singing query. Experiments have been conducted to assess the overall performance and examine the effects of various parameters introduced in the proposed method.
\end{abstract}

\section{KEYWORDS}

Music Melody Plagiarism, Music Melody Retrieval, Subsequence Matching, Warping Time Series Join

\section{INTRODUCTION}

No matter how the modern life changes, music appreciation is always one of the easy and affordable ways to slacken the strain of our daily lives. Nowadays, common users can acquire musical contents easily anywhere and anytime with the Internet. The large demands from the vast number of music-goers have been driving the fast growth of the market of music production, inspiring potential music composers plunging into the realm of music creation. To fit the general tastes of worldwide audience and meet the high production rate requested by music production companies, composers often habitually take some existing popular music pieces as the inspiring sources for faster composition. Occasionally, composers may intentionally or unintentionally mimic some pieces of melodies from others' work, thus may causing the illegal plagiarism of music arts. We all are aware of that illegal piracy of musical intellectual properties can severely obstruct the development of music creation and marketing business. Finding the plagiarized pieces among the fast-growing number of music contents is not trivial. Manual examination costs very high in both time and human power. To prevent the music plagiarism and to keep the prosperous production of the legal music arts, an efficient way to detect automatically the piracy among created music contents becomes highly desirable. 
One major challenge of AMMPD is that the plagiarised pieces can appear at any place in a music melody. Exhaustive searching by matching all subsegments of two suspicious music melodies is very time consuming. Additionally, the AMMPD may also suffers from the problem of inexact matching due to some tonal variations on the plagiarized melody pieces. Hence, the AMMPD demands an efficient and effective solution to locate similar melody pieces in two music melodies. The objective of our work presented in this paper is to propose such a solution for developing an AMMPD system.

\section{RELATED WORK}

According to our literature survey, we have not found any related study on the issues of AMMPD. Most prior arts that have been published are applications relating to retrieval of musical contents. For example, the query by singing/humming (QBSH) are one of the typical topics $[1,2$, 3]. The problem of QBSH is different from that of AMMPD. One major difference is that the query input in the QBSH has a clear pair of end points (both the starting point and the ending point) for matching with the partial pieces of each music content in the database. However, for the problem of AMMPD, since we are not aware of where the plagiarised pieces may appear in both the query input and a database content, detecting and locating the plagiarised pieces are thus more cumbersome. Particularly, the possible existence of multiple plagiarised pieces inside two suspicious melodies further complicates the problem.

To address the QBSH problem, the Dynamic Time Warping (DTW) [4] algorithm is a common way to do the nonlinear sequence matching. Athitsos et al. [5] proposed a DTW-based method for approximate subsequence matching of time series. Their method is applicable only to the matching between the whole query input an subsequences of a sequence in the database. For the AMMPD problem that requires intensive subsequence matching between the query input and the database sequence, their method is not feasible. Some methods underlain by the longest common subsequences algorithm [6] are also not suitable because they deal with only noisy-free sequences. That is, the matching between subsequences must be exact matching, instead of the inexact matching required by the tonal features of music audio. Lin et al. [7] presented a method using the rough longest common subsequence algorithm to address the music retrieval problem which is similar to the QBSH. Not aiming at the problem of music processing, Chen et al. proposed a warp time series join (WTSJ) algorithm to find the similar subsegments between two motion sequences as the best part for fusing the two sequences. Though the WTSJ algorithm can locate similar pieces in two motion sequences, the algorithm may suffer from some problems, such as poor alignment and over-segmentation of similar pieces, due to the weak capability in handling noisy elements in sequences.

In this paper, we address the issues of AMMPD by a 2-D spatial-domain approach, rather than the 1-D temporal-domain approach that underlies the methods mentioned above. The proposed method tackles the subsequence matching problem for AMMPD by the path exploration method over a binary mask, which incorporates some image-based techniques, such as gap filling, block partitioning, connected path finding, and polyline approximation of distorted paths. Some tricky designs have been incorporated in the proposed method to handle the noisy pitch features of music audio. The proposed method can not only locate all possible plagiarised subsegments in two suspicious melodies, but also retrieve music contents that contain the melody pieces similar to the input query of a music clip. Hence, the proposed method is feasible for both the AMMPD problem and the QBSH problem. 


\section{THE PROPOSED METHOD}

\subsection{Overview}

Fig. 1 shows the process flow of the proposed AMMPD method. Given two suspicious music melodies, the pitch extraction module extracts the pitch vectors, $Q=\left[q(1), q(2), \ldots, q\left(F_{1}\right)\right]$ and $D=\left[d(1), d(2), \ldots, d\left(F_{2}\right)\right]$, of the two melodies by a pitch tracking method. Since the two pitch vectors may contain some noisy pitch elements, we smoothen them by applying the median filter of the window size $m$. From the two smoothed pitch vectors, the pairwise distances between the pitch elements in the pitch vectors are calculated to derive a local distance matrix. By setting a threshold $\varepsilon$, we binarized this local distance matrix as a binary 2-D mask $M$ where an entry $M(i, j)=1$ indicates a possible correspondence between the pitch pair $(q(i), d(j))$ in the two melodies $Q$ and $D$. In contrast, an entry of 0 on the mask $M$ means a dissimilar pitch pair in the two melodies. Hence, a rectangular block containing contiguous entries of 1's on $M$ actually identifies preliminarily a potential similar subsegments in the two sequences. One important task for the subsequent processing is to partition the mask into several rectangular blocks that reveals the potential plagiarised subsegments. To avoid the over-partitioning of the mask due to the unexpected pairwise pitch dissimilarity caused by some singular pitch elements, we fill the minor gaps, whose sizes are defined by a parameter $g$, between blocks before the mask partitioning. After the minor gap filling, the binary mask $M$ is partitioned by finding vertical and horizontal gaps (consecutive entries of 0 along vertical and horizontal directions) between the blocks. Note that the partitioning is repeatedly done on every block until no vertical or horizontal gap appears on the block. After the partitioning, all paths of connected 1-entries inside each block are explored. Meanwhile, the detailed correspondences between the pitch elements along the path are also derived. Afterwards, multiple overlapping paths in each block can be ranked according to the derived detailed correspondences. An explored path may also have some distorted sections that reveal unreasonable many-to-one or one-to-many correspondences between pitch elements. These many-to-one and one-to-many correspondences alone the path can usually be found on the sections that are horizontal/near-horizontal or vertical/near-vertical. Hence, we remove these distorted sections by approximating the path with multiple polylines and removing those polylines that are horizontal/near-horizontal or vertical/near-vertical. Eventually, those paths that are too short to identify plagiarised subsegments are eliminated.

\subsection{Pitch Vector Extraction of Music Audio}

A digitized music data contains a sequence of audio data sampled at a fixed rate such as $8 \mathrm{KHz}$ or $16 \mathrm{KHz}$. The data sequence is then partitioned into frames with each containing a fixed number of samples and overlapping partially with its neighboring frames. Applying the pitch tracking method [8] to each frame, we can estimate the fundamental frequency of the audio samples as the pitch of the frame. Given a fundamental frequency $f$, the pitch corresponds to a semitone $n=69+12 \log _{2}(f / 440)$ which ranges normally over $35 \sim 72$ for male sounds and $45 \sim 83$ for female sounds. Therefore, an music audio of $F$ frames can get a pitch vector of $F$ semitones to characterize the tonal feature.

Unfortunately, the estimated fundamental frequency of each frame is not always robust under the disturbance of noisy audio signal. The output pitch vector may contain some noisy semitones which can incur undesired effects on matching music melodies. To reduce the effect of the noisy semitones, we perform the median filtering on the elements of the pitch vector to remove some singular noise pitch elements. However, for some continuous occurrences of noisy pitch elements, other methods is still necessary to remedy the possible side effects. We will present a way in later descriptions of the proposed method. 


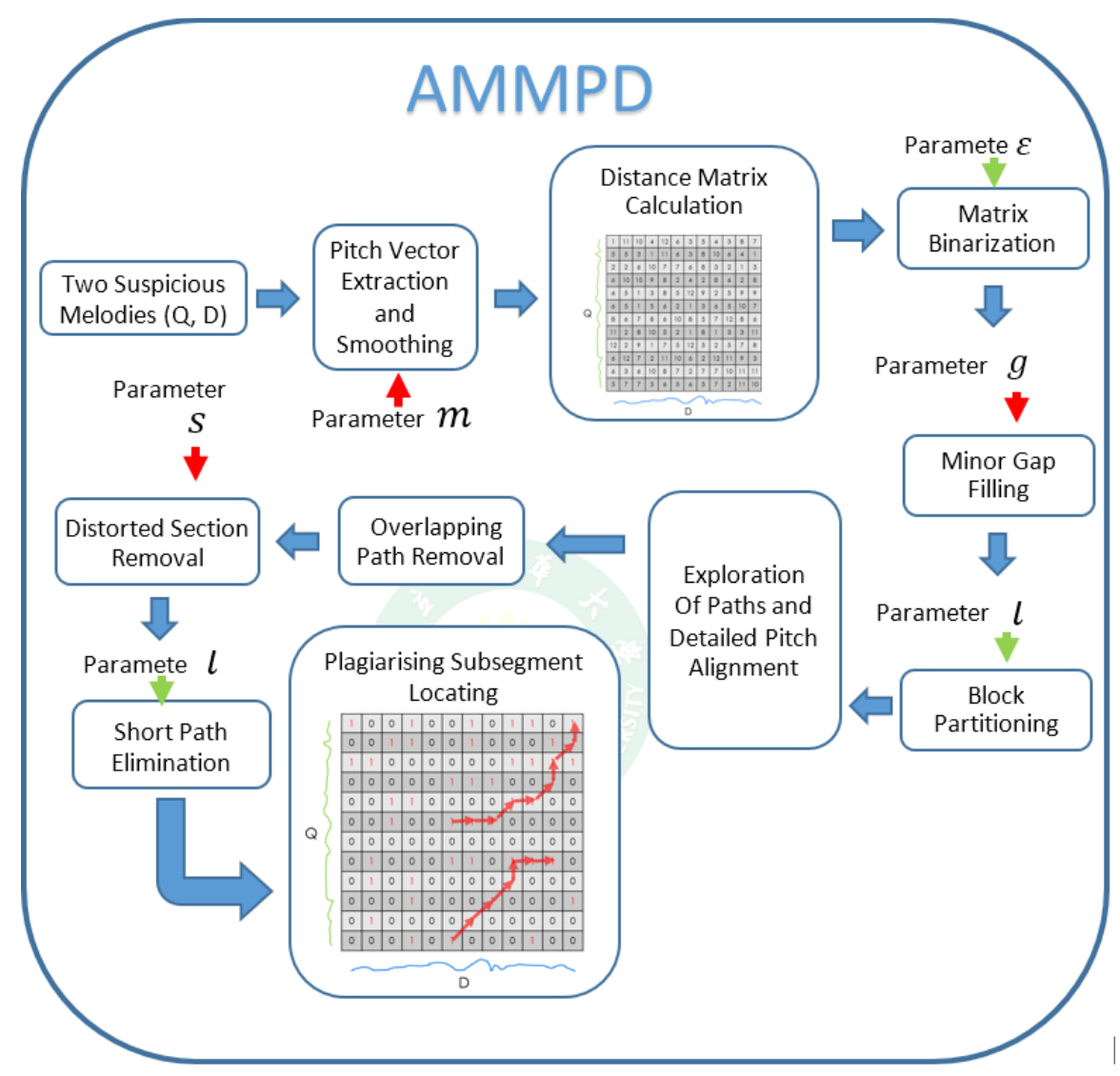

Figure 1: The process flow of the proposed AMMPD method.

\subsection{Distance Matrix Calculation and Binarization}

Let $Q=\left[q(1), q(2), \ldots, q\left(F_{1}\right)\right]^{T}$ and $D=\left[d(1), d(2), \ldots, d\left(F_{2}\right)\right]^{T}$ be the pitch vectors of two suspicious music melodies. Computing the pairwise distances between the pitch elements in the two vectors lead to a 2 -D distance matrix $\mathbb{D}(i, j)=|q(i)-d(j)|$ for $1 \leq i \leq F_{1}$ and $1 \leq j \leq F_{2}$. The entries in the matrix $\mathbb{D}$ can be quantized into similar indications and dissimilar indications by specifying a distance threshold $\varepsilon$ which defines the maximal dissimilarities allowed between the pitch elements of two plagiarised pieces. In other words, we can derive a 2-D binary mask $M$ by

$$
\mathrm{M}(\mathrm{i}, \mathrm{j})=\left\{\begin{array}{rr}
1, & \text { if } \mathbb{D}(i, j)<\varepsilon \\
0, & \text { otherwise }
\end{array}\right.
$$

where an an entry $M(i, j)$ of 1 indicates a similar pair of pitch elements $(q(i), d(j))$ in the two suspicious melodies. On the contrary, an entry of 0 implies that the pitch elements $q(i)$ and $d(j)$ are too different to be two corresponding pitches in plagiarised pieces. Fig. 2 shows an example of $\mathbb{D}$ and $M$ with the threshold $\varepsilon$ of 4 . 


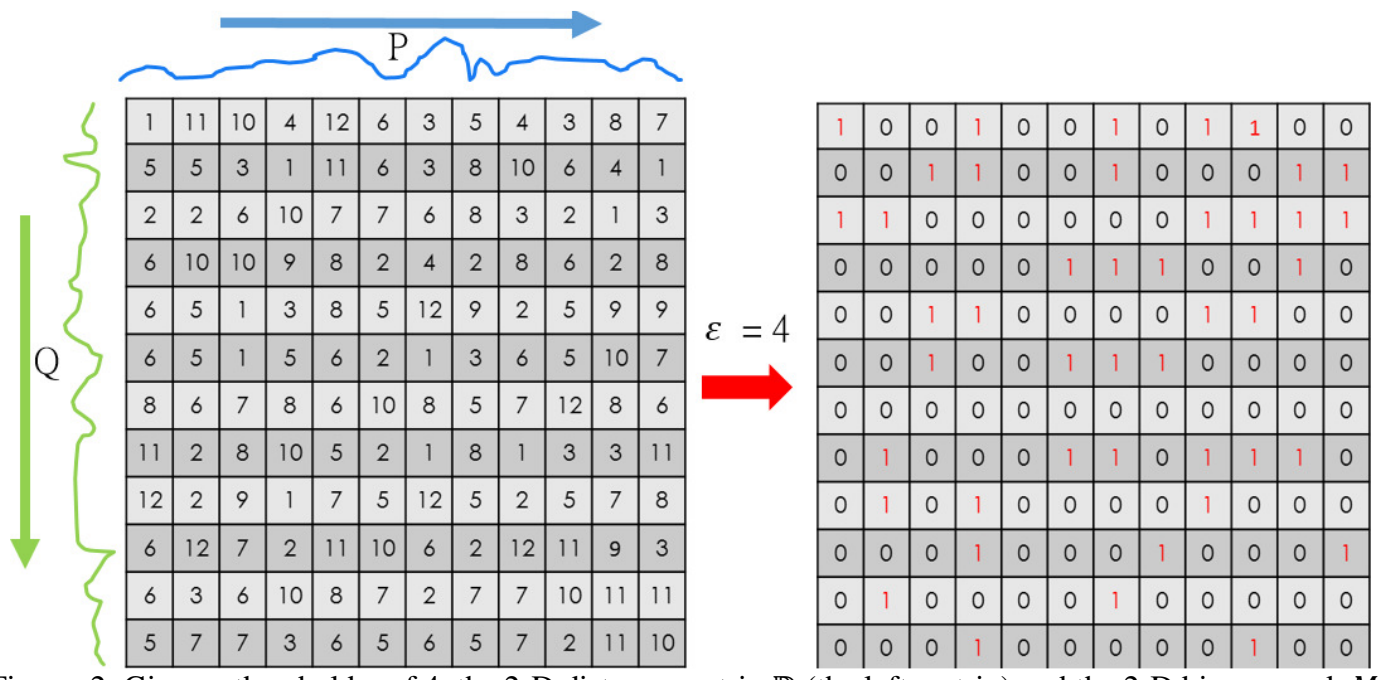

Figure 2: Given a threshold $\varepsilon$ of 4 , the 2-D distance matrix $\mathbb{D}$ (the left matrix) and the 2-D binary mask $M$ (the right matrix) derived from two pitch vectors $Q$ and $D$.

\subsection{Minor Gap Filling}

On the derived binary mask, we can find some entries of 1 connected as many paths. A path may start from a certain entry $M(i, j)$ and stop at another $M\left(i^{\prime}, j^{\prime}\right)$. We denote such a path as $o\left(u(i, j) \Rightarrow u^{\prime}\left(i^{\prime}, j^{\prime}\right)\right)$, where $u$ and $u^{\prime}$ are called nodes. Note that the entry of the mask on a node is always 1 . Besides, a node $(i, j)$ can have at most only three possible preceding nodes, i.e., $(i-1, j),(i, j-1),(i-1, j-1)$. For convenience, we also use the notations, $u . i$ and $u . j$, to represent the row index and column index of the node $u$, respectively. In physical meaning, each path actually corresponds a pair of potential plagiarised subsegments $q\left(i: i^{\prime}\right)$ and $d\left(j: j^{\prime}\right)$ in the two melodies, respectively. Hence, one important task of the proposed AMMPD method is to explore all potential connected paths over the binary mask $M$.

Since every path always breaks at an entry of 0 on $M$, the exploration of the paths can be done by finding rectangular blocks separated by horizontal and vertical gaps formed by 0 -entries. However, the separation of rectangular blocks are prone to the gaps formed by some singular nodes $(i, j)$ 's with larger pairwise distances $\mathbb{D}(i, j)$ 's incurred from noisy pitch elements. Like the binary mask shown in Fig. 3 (a), the red areas and white areas contain respectively 1-entries and 0 -entries. A small gap appearing on the singular node of entry-0 highlighted with the blue circle breaks a path. As a result, the broken path may cut the true plagiarised subsegments, as shown in Fig. 3 (b), into shorter ones.

To avoid the bad break of paths, we perform a gap filling process to bridge the paths separated by the singular nodes. This filling process fills those entries between a node and the nearest node along the horizontal, the vertical, and the diagonal direction according to the following three rules:

- Horizontal Filling: $M(i+k, j)=1$ for $1 \leq k \leq k^{*}-1 \leq g$ if $M(i+k, j)=0 \wedge M(i+$ $\left.k^{*}, j\right)=1$

- Vertical Filling: $M(i, j+k)=1$ for $1 \leq k \leq k^{*}-1 \leq g$ if $M(i, j+k)=0 \wedge M(i, j+$ $\left.k^{*}\right)=1$

- Diagonal Filling: $M(i+k, j+k)=1$ for $1 \leq k \leq k^{*}-1 \leq g$ if $M(i+k, j+k)=0 \wedge$ $M\left(i+k^{*}, j+k^{*}\right)=1$; 
Note that the parameter $g$ is set as the largest gap width allowed for the gap filling. The gaps with the width larger than $g$ will not be filled. Taking the case in Fig. 3 (a) as an example, the resultant path explored after the gap filling is the one shown in Fig. 3 (b).

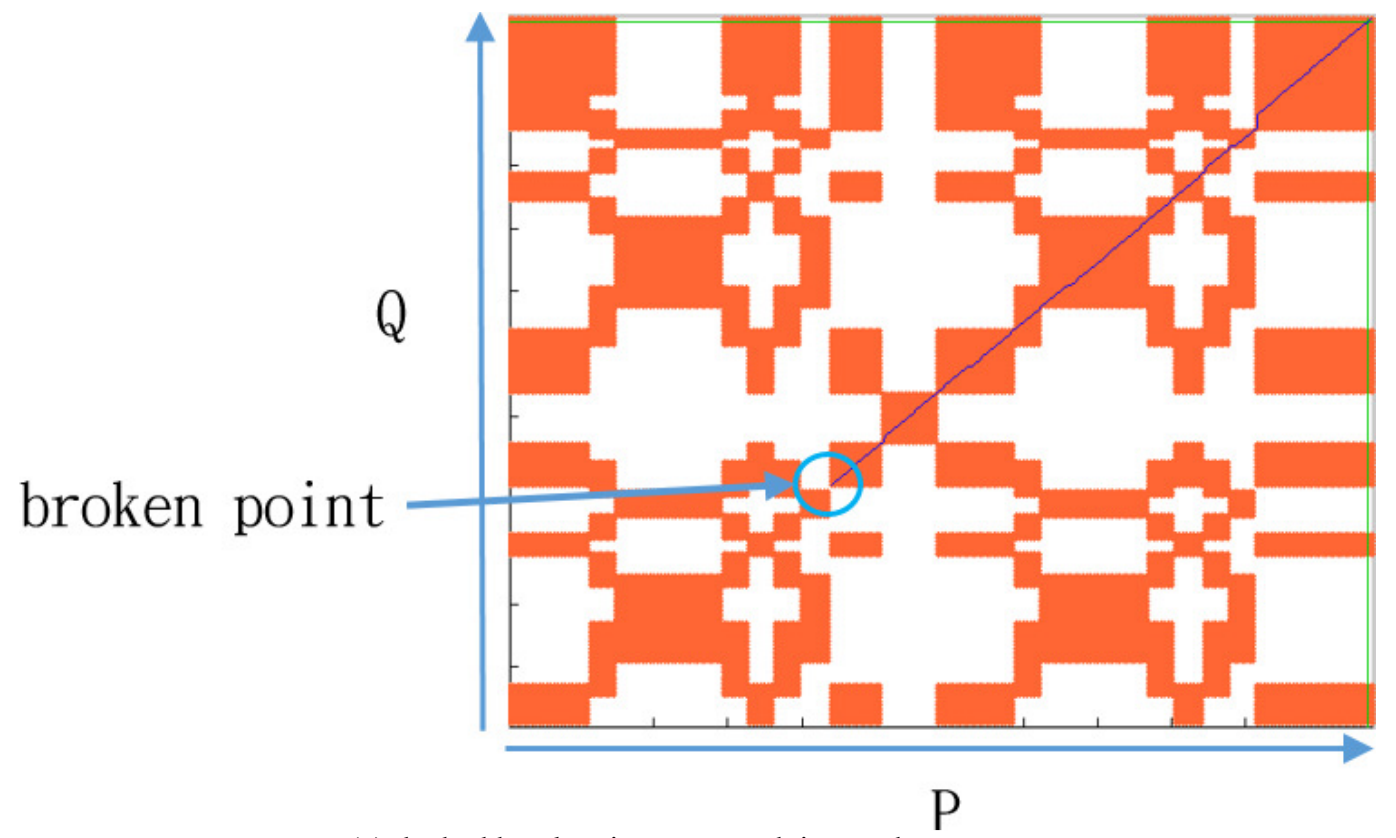

(a) the bad break point cuts a path into a shorter one.

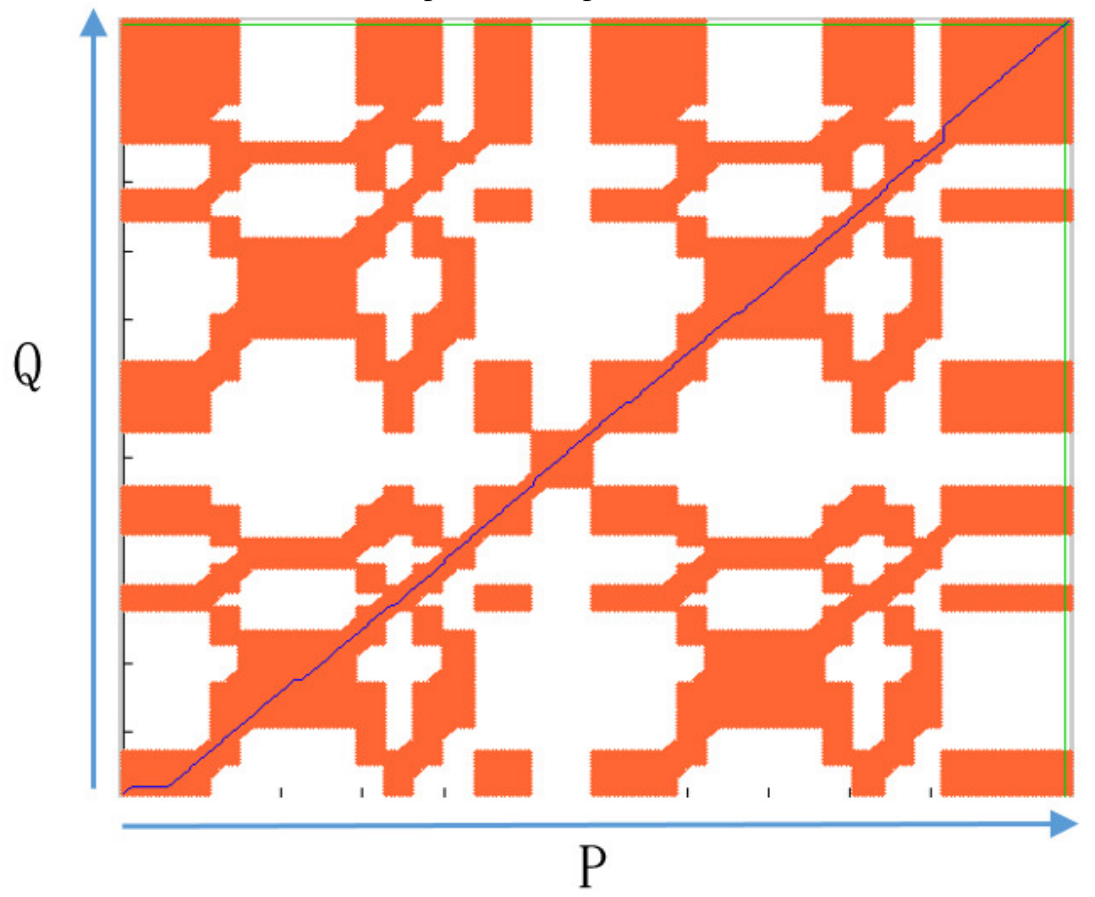

(b) the longer path formed in the block without the bad break point.

Figure 3: Improper partitioning of blocks of caused by noisy pitch elements. 


\subsection{Block Partitioning}

Some paths of connected 1-entries are not long enough to identify a pair of plagiarized subsegments in two melodies. To fast remove these paths before exploring the connected nodes in them, we exploit a recursive block partitioning procedure on the binary mask to extract all potential rectangular blocks that enclose the paths. If the size of a block is too small, then all paths inside the block can be ignored directly with having to explore the connected nodes along them. The steps of the recursive block partitioning procedure Partition () are listed in Procedure 1 . The blocks on the mask $M$ can be derived by making a call to $\operatorname{Partition}\left(1, F_{1}, 1, F_{2}\right)$, where $F_{1}$ and $F_{2}$ are the height and width of $M$, respectively.

In the set of blocks obtained from the partitioning procedure, any block $B$ can be discarded if width $(B)<l$ or height $(B)<l$, where $l$ specifies the smallest length of any plagiarised subsegment. This would save the efforts to explore all connected nodes along the paths inside the block $B$.

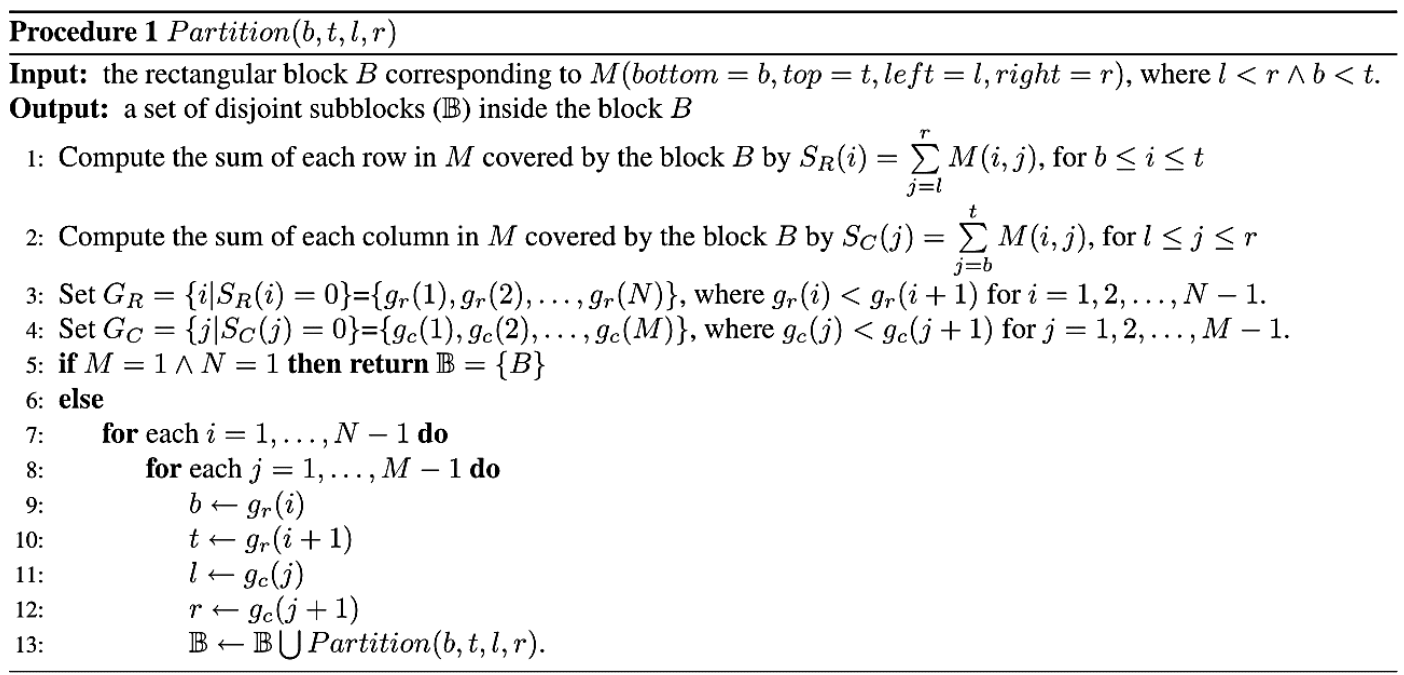

\subsection{Path Exploration by Finding Detailed Alignment of Pitch Elements}

Once the blocks inside the binary mask are available, the paths inside each block need to be explored. A path is a sequence of connected nodes. The starting node $(i, j)$ is an open node that has no preceding nodes, i.e., $M(i-1, j)=M(i, j-1)=M(i-1, j-1)=0$. In contrast, the stopping node $(i, j)$ of a path is a close node that has no succeeding nodes, i.e., $M(i+1, j)=$ $M(i, j+1)=M(i+1 . j+1)=0$. Any node that is neither an open node nor a close node is an intermediate node. Hence, all nodes in a block actually form a directed acyclic graph (DAG). Every node corresponds to a graph node. A graph node $(i, j)$ connects only to its preceding nodes $(i-1, j),(i, j-1)$, and $(i-1, j-1)$ if the three nodes of 1-entries do exist (nodes do not exist for 0 -entries). With the constructed DAG, all paths from an open node $\left(u_{o}\right)$ to a close node $\left(u_{c}\right)$ can be found by the procedure Explore $\left(u_{o}, u_{c}\right)$ listed in Procedure 2. Some notations are introduced in the procedure. First, predecessor $(u)$ and $\operatorname{successor}(u)$ mean the direct succeeding nodes and the direct preceding nodes of a node $u$, respectively. The notation $c(u \triangleright$ $u^{\prime}$ ) denotes a connection connecting the two nodes $u$ and $u^{\prime}$. The operator $c \oplus o$ denotes the concatenation of a connection $c$ and a path $o$. To explore all paths inside a block, we just need to find all open nodes and close nodes and then make a call to the procedure Explore $\left(u_{o}, u_{c}\right)$ for each pair of open node $u_{o}$ and close node $u_{c}$. 
There might exist relations of dominance among the paths explored by theExplore () procedure. A path $o(u \Rightarrow v)$ dominates another path $o^{\prime}\left(u^{\prime} \Rightarrow v^{\prime}\right)$ if $\left(u . i<=u^{\prime} . i\right) \wedge\left(u . j<=u^{\prime} . j\right) \wedge(v . i>$ $\left.v^{\prime} . i\right) \wedge\left(v . j>v^{\prime} . j\right)$. The physical meaning of the dominance is that the plagiarised subsegment implied by the path $o$ contains that implied by the path $o^{\prime}$. In case that a path $o$ dominates another path $o^{\prime}$ in the set of explored paths, then $o^{\prime}$ is removed from the path set because it is redundant.

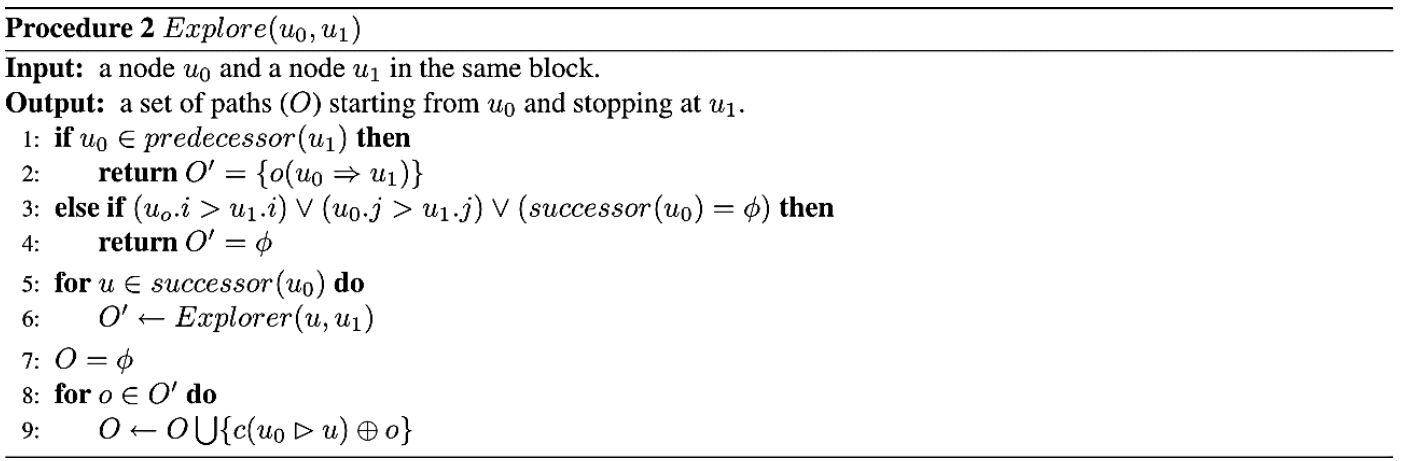

\subsection{Overlapping Path Removal}

After removing all dominated paths from the set of explored paths, some paths may overlap with others. The overlapping paths may share some connected nodes, but do not dominate each other. As shown in Fig. 4, three paths share a large portion of the paths. However, these three paths identify three different pairs of plagiarized subsegments in the two melodies. Similarly, the three pairs of plagiarized subsegments also have a large part in common and thus may cause redundant detection of plagiarism. One reasonable action is to choose the best one among them. To do this, we need to define a criterion for assessing the goodness of a path. As every explored path contains a sequence of connected nodes $u(i, j)$, with each meaning a corresponding pair of pitch elements $(q(i), d(j))$. Along the path, every two neighboring nodes must be a node $u$ and a successor node of the node $u$. Hence, a path can consist of three kinds of connections for any two neighboring nodes, including vertical, horizontal, and diagonal node connections. A vertical node connection $(i-1, j) \triangleright(i, j)$ implies a two-to-one correspondence of pitch elements, i.e., $\{q(i-1), q(i)\} \rightarrow\{d(j)\}$. A vertical node connection $(i, j-1) \triangleright(i, j)$ implies a one-to-two correspondence of pitch elements, i.e., $\{q(i)\} \rightarrow\{d(j-1), d(j)\}$. A diagonal node connection $(i-1, j-1) \triangleright(i, j)$ implies a one-to-one correspondence, i.e., $\{q(i-1)\} \rightarrow\{d(j-1)\}$ and $\{q(i)\} \rightarrow\{d(j)\}$. Obviously, the one-to-one correspondence gives a better explanation to the correspondence between elements of plagiarised subsegments. Therefore, a feasible index to assess the goodness of a path $o$ the number of diagonal node connections, say $\operatorname{Diag}(o)$, along the path. Based on the criterion, Diag $(o)$, the procedure to remove those overlapping paths with lower values of $\operatorname{Diag}(o)$ is designed as the RemoveOverlappingPaths $(O, l)$ listed in Procedure 3.

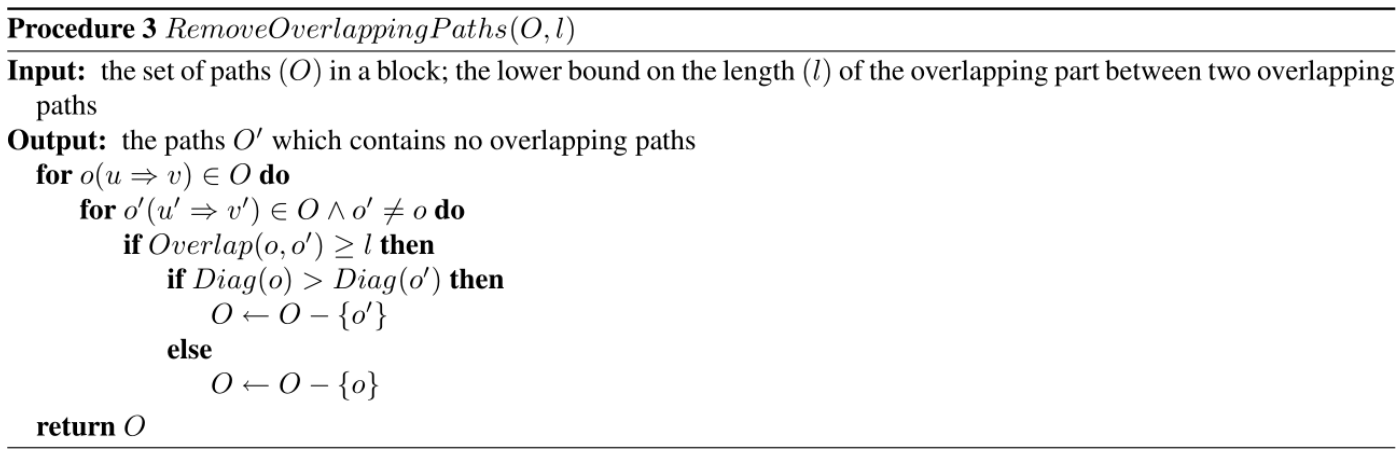




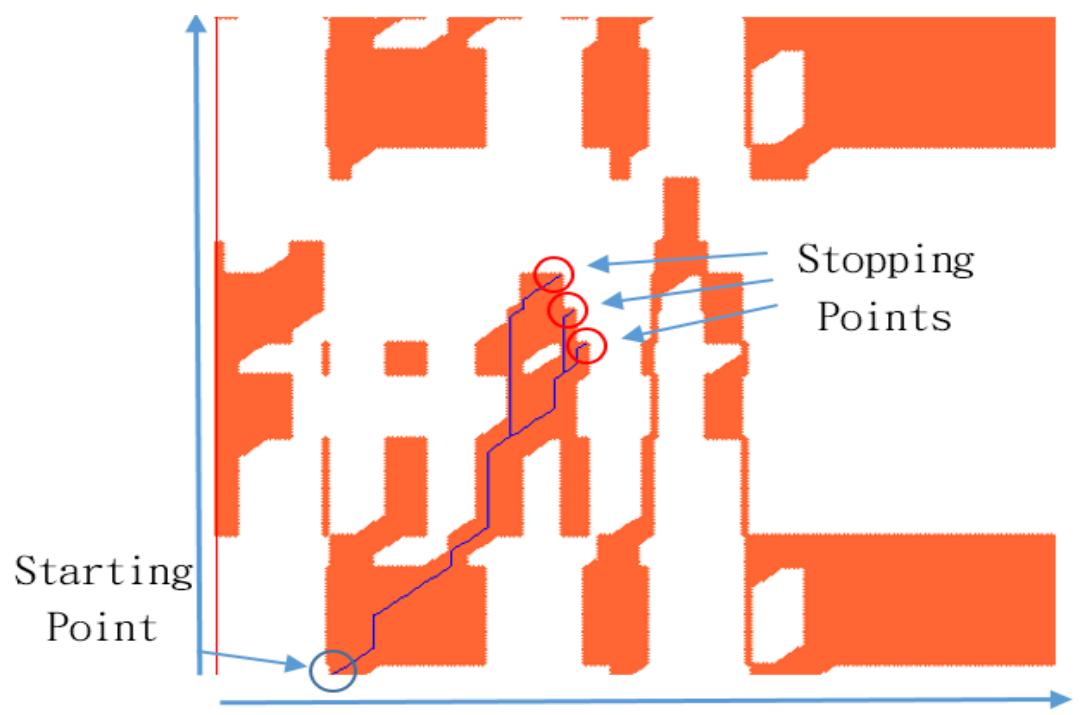

Figure 4: Example cases of overlapped paths.

\subsection{Removal of Distorted Sections in Eligible Paths}

Every path that remains after the overlapping path removal is an eligible path for identifying plagiarised subsegments between the two suspicious melodies. However, there may still exist one kind of oddness on the path. Taking Fig. 5 as an example, we can see that some nodes have oneto-many or many-to-one correspondences, as highlighted with blue circles. These one-to-many correspondences or many-to-one correspondences would cause longer sections of vertical node connections or horizontal node connections inside the path. We refer to this kind of sections as distorted sections which may not be appropriate to identify the pieces in plagiarised subsegments. Namely, we should remove these distorted sections from a path to prevent false detection of plagiarised pieces.

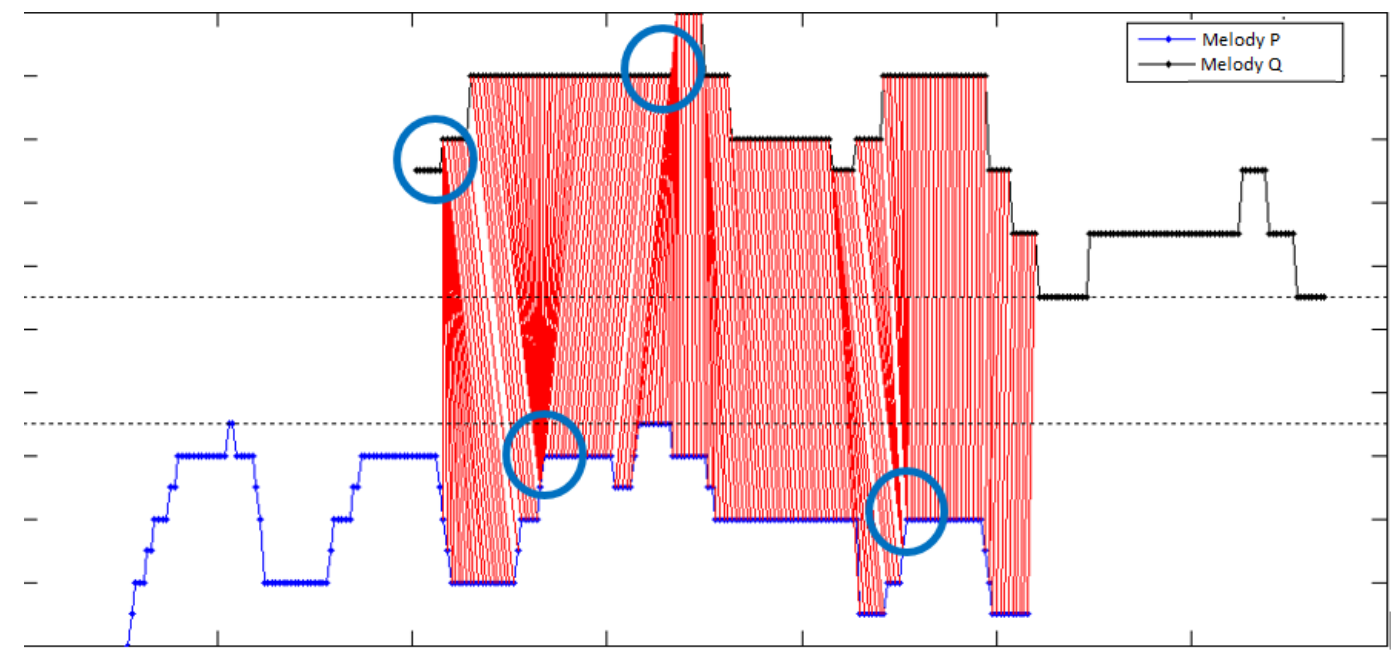

Figure 5: The alignment of the pitch elements between two melodies may contain unreasonable one-tomany and many-to-one correspondences.

As shown in Fig. 6(a), a long path may comprise several horizontal and vertical sections highlighted with circles. The distorted sections in blue circles are longer, while those in red circles are shorter. For the path illustrated in Fig.6(a), the true section corresponding to the 
plagiarised subsegments in the two melodies is the section enclosed by the large blue rectangle. To extract the non-distorted sections from an eligible path, our method first approximates the path with a polyline. Afterwards, the horizontal/vertical and near-horizontal/near-vertical segments in the polyline are removed. In doing so, a segment $\bar{o}$ in the polyline is removed if $|\operatorname{slope}(\bar{o})-1|>$ $\delta$, where slope $(\bar{o})$ is the slope of the segment $\bar{o}$ and $\delta$ is the threshold specified for the removal. Fig. 6(b) shows the result after applying the method to the path in Fig. 6(a). The result demonstrates that this method works fine to extract the desired non-distorted section from the path.

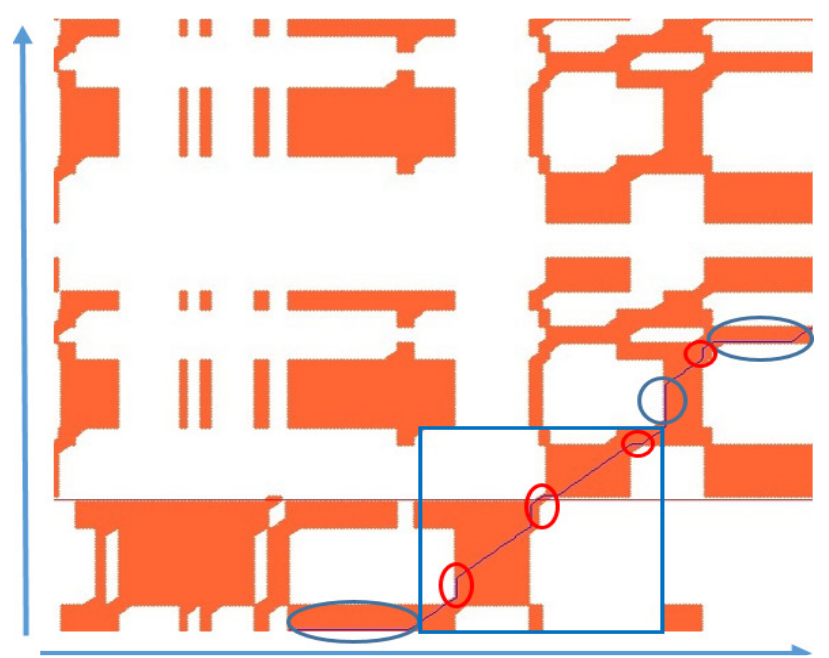

(a) The path with distorted sections

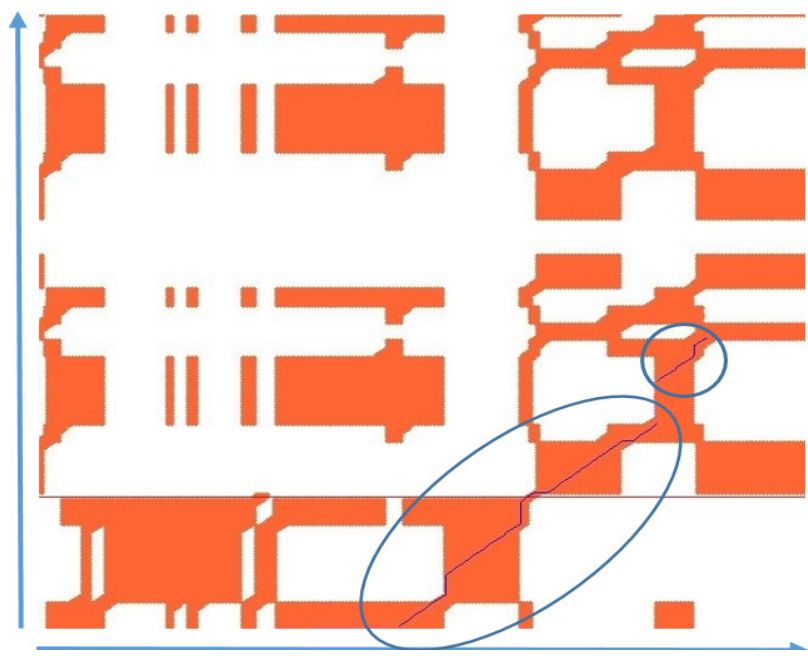

(b) The segmented paths after removing distorted sections

Figure 6: Examples of distorted sections in a path.

After removing the distorted sections in a path, some paths may become shorter and even dominated by others. These paths should be purged, too. According to the specified parameter $l$ in block partitioning, we discard those paths with the lengths smaller than $l$. Additionally, as done at the end of path exploration, the path dominated by others are also discarded. 


\section{EXPERIMENTAL RESULTS}

\subsection{Data Set and Types of Experiments}

Several experiments are conducted to evaluate the performance of the proposed method. We use the MIR-QBSH data set created by Chang et al [9] for the experiments. The data set contains 48 ground-truth MIDI files and 4431 singing/humming clips from about 195 subjects. Since the songs in the data set does not contain the plagiarising pieces, we randomly partition the 48 MIDIs into 16 groups, with each having three. Then, the three MIDIs in each group are concatenated as on MIDI to get 16 concatenated MIDI songs. Besides, we also randomly compose 100 concatenated voiced songs from 300 signing/humming clips which enclose all melodies of the 48 MIDIs sung/hummed by at least 6 different subjects. Each concatenated voiced song also has three different signing/humming clips. Three types of experiments are conducted on the composed data set. Type A experiments use one concatenated voiced MIDI song as the input and search the possible plagiarising pieces in all concatenated voiced songs of the database. Type B experiments use one single MIDI song (not a concatenated MIDI song) as the input and search the possible plagiarism in all concatenated voiced songs of the database. Finally, Type C experiments use a single MIDI song as the input and search the similar single signing/humming clips. The indices for performance evaluation are the precision rate and the recall rate. A correct detection of a plagiarising subsegment requires that the following condition hold true for the detected subsegment $d$ and the true plagiarising subsegments in two melodies $a$ and $b$ :

$$
\left(\frac{\operatorname{len}(d \cap a)}{\operatorname{len}(a)} \geq \frac{1}{3}\right) \wedge\left(\frac{\operatorname{len}(d \cap b)}{\operatorname{len}(b)} \geq \frac{1}{3}\right)
$$

where $\operatorname{len}(s)$ denotes the length of a subsegment $s$.

\subsection{Overall Performance Evaluation}

The performance of the proposed method is evaluated from the results of the three types of experiments. Table 1 lists the averaged precision rate and recall rate for these three types of experiments. The achieved precision rate is about $84 \%$ and the recall rate is about $63 \%$. Because of the inaccuracy of pitch extraction, the recall rates are much lower than the precision rates. The noisy pitch vectors can cause the mismatch of many subsegments with similar melodies in suspicious music audio. The median filtering and block gap filling proposed in the method seem take only minor effect in handling the noisy pitch features. Particularly, the query input, which is a MIDI clip, and the database targets, which are humming/singing clips, are different audio source which reveal different audio characteristics. Moreover, as the pitch of a humming/singing clip varies with different subjects, the pitch vectors extracted from the audio clips of the same melody still may differ significantly for different subjects.

Table 1. The overall precision rates and recall rates for the three types of experiments. $X_{p}$ and $X_{r}$ denote respectively the precision rate and the recall rate of Type $X$ experiments.

\begin{tabular}{|c|c|c|c|c|c|}
\hline $\mathbf{A}_{\mathrm{p}}(\%)$ & $\mathbf{A}_{\mathbf{r}}(\%)$ & $\mathbf{B}_{\mathrm{p}}(\%)$ & $\mathrm{B}_{\mathbf{r}}(\%)$ & $\mathbf{C}_{\mathrm{p}}(\%)$ & $\mathbf{C}_{\mathbf{r}}(\%)$ \\
\hline 82 & 64 & 83 & 63 & 87 & 63 \\
\hline
\end{tabular}

\subsection{Examining the Effects of Different Parameters}

There are some parameters introduced in the proposed method, including

- $\varepsilon$ : the upper bound on the pairwise distances of pitch elements in two suspicious melodies; 
- $l$ : the lower bound on the length of an eligible candidate path for locating the plagiarising subsegments in two melodies;

- $g$ : the parameter defining the upper bound on the minor gaps to be filled between blocks on the binary mask $M$;

- $\delta$ : the slope parameter used for removing the distorted sections along an eligible path.

In the following, the effects of these parameters are examined and discussed with the experimental results.

\subsubsection{The Effect of $\varepsilon$}

The parameter $\varepsilon$ affects mainly the number of blocks formed on the binary mask $M$. Table 2 shows the precision rates and recall rates corresponding to the different settings of $\varepsilon$, i.e., $\varepsilon=0,1$, and 2. All other parameters are set as $m=5, l=150, g=3$, and $\delta=0.4$. The extremal case of $\varepsilon=0$ causes that two suspicious melodies are detected as plagiarism only when they are exactly identical. The results show that a higher setting can increase the recall rate because it allows larger pitch differences between two plagiarising subsegments. However, loosened condition of plagiarism may also increases the number of false alarms and thus decreases the precision rate.

Table 2: The precision rates and recall rates for different setting of $\varepsilon . X_{p}$ and $X_{r}$ denote respectively the precision rate and the recall rate of Type $X$ experiments.

\begin{tabular}{|r|r|r|r|r|r|r|}
\hline$\varepsilon$ & $\mathbf{A}_{\mathrm{p}}(\boldsymbol{\%})$ & $\mathbf{A}_{\boldsymbol{r}}(\boldsymbol{\%})$ & $\mathrm{B}_{\mathrm{p}}(\boldsymbol{\%})$ & $\mathrm{B}_{\mathrm{r}}(\boldsymbol{\%})$ & $\mathrm{C}_{\mathrm{p}}(\%)$ & $\mathrm{C}_{\mathbf{r}}(\%)$ \\
\hline 0 & 100 & 0 & 100 & 0 & 100 & 0 \\
\hline 1 & 82 & 64 & 83 & 63 & 87 & 63 \\
\hline 2 & 27 & 80 & 31 & 79 & 35 & 79 \\
\hline
\end{tabular}

\subsubsection{The Effect of $l$}

The parameter $l$ specifies at least how long a true plagiarisinig subsegement must be. We test $l$ with the different values $120,130,140,150$, and 160 . Note that a length of 150 pitch elements is about $150 * 0.032(=4.8)$ seconds. Table 3 lists the precision rates and recall rates for these different settings. The larger the $l$, the longer the plagiarising subsegments must be. Therefore, some shorter plagiarising subsegments are more likely to be missed and the recall rate tends to decrease. On the other hand, some shorter false alarms can be avoided and thus the precision rate tends to increase.

Table 3: The precision rates and recall rates for different setting of $l$.

\begin{tabular}{|c|c|c|c|c|c|c|}
\hline $\boldsymbol{l}$ & $\mathbf{A}_{\mathrm{p}}(\%)$ & $\mathbf{A}_{\mathbf{r}}(\%)$ & $\mathrm{B}_{\mathbf{p}}(\%)$ & $\mathbf{B}_{\mathbf{r}}(\%)$ & $\mathrm{C}_{\mathrm{p}}(\%)$ & $\mathbf{C}_{\mathbf{r}}(\%)$ \\
\hline 120 & 33 & 76 & 36 & 76 & 41 & 76 \\
\hline 130 & 51 & 74 & 54 & 73 & 59 & 72 \\
\hline 140 & 67 & 69 & 70 & 69 & 74 & 69 \\
\hline 150 & 82 & 64 & 83 & 63 & 87 & 63 \\
\hline 160 & 89 & 60 & 90 & 59 & 92 & 59 \\
\hline
\end{tabular}




\subsubsection{The Effect of $g$}

The parameter $g$ defines the upper bound on the minor gaps between blocks on the binary mask $M$. To prevent the binary mask being over-partitioned into many small blocks, the proposed method fills the minor gaps between blocks. A larger value of $g$ causes more blocks connected through the gap filling process, thus usually resulting in more detected plagiarising subsegments. Though the increased detection of plagiarism improves the recall rate, the precision rate may decrease due to the increased number of false alarms. The precision rates and the recall rates for different settings of $g$ are shown in Table 4. The results show that an appropriate value for the parameter $g$ ranges between 3 and 5 .

Table 4: The precision rates and recall rates for different setting of $g$.

\begin{tabular}{|c|c|c|c|c|c|c|}
\hline$g$ & $\mathbf{A}_{p}(\%)$ & $\mathbf{A}_{\mathbf{r}}(\%)$ & $\mathbf{B}_{\mathrm{p}}(\%)$ & $\mathbf{B}_{r}(\%)$ & $\mathrm{C}_{\mathrm{p}}(\%)$ & $\mathrm{C}_{\mathrm{r}}(\%)$ \\
\hline 0 & 88 & 38 & 88 & 38 & 90 & 38 \\
\hline 1 & 85 & 49 & 86 & 49 & 89 & 49 \\
\hline 2 & 84 & 54 & 86 & 54 & 89 & 54 \\
\hline 3 & 82 & 64 & 83 & 63 & 87 & 63 \\
\hline 4 & 79 & 69 & 80 & 69 & 86 & 69 \\
\hline 5 & 72 & 74 & 74 & 73 & 81 & 73 \\
\hline 6 & 66 & 76 & 70 & 75 & 77 & 74 \\
\hline 7 & 59 & 76 & 63 & 75 & 71 & 75 \\
\hline
\end{tabular}

\subsubsection{The Effect of $\delta$}

The parameter $\delta$ specifies the deviation of the slope of a path from a $45^{\circ}$ diagonal line. A large deviation indicate that the path is a distorted path which contains improper alignment of pitch elements and should be discarded. Hence we experiment the proposed method with the settings of $\delta$ ranging between 0.1 and 0.8 . The smaller the $\delta$ is, the better element correspondences an eligible path may derive, meaning that a better precision rate we may achieve. However, the side effect is that some true plagiarising subsegments with slightly distorted correspondences may be missed. Table 5 shows the precision rates and recall rates for the different settings of $\delta$.

Table 5: The precision rates and recall rates for different setting of $\delta$.

\begin{tabular}{|c|c|c|c|c|c|c|}
\hline 8 & $\mathbf{A}_{\mathrm{p}}(\%)$ & $\mathbf{A}_{\mathbf{r}}(\%)$ & $\mathbf{B}_{p}(\%)$ & $\mathrm{B}_{\mathrm{r}}(\%)$ & $C_{p}(\%)$ & $\mathrm{C}_{\mathrm{r}}(\%)$ \\
\hline 0.1 & 89 & 41 & 88 & 41 & 89 & 41 \\
\hline 0.2 & 88 & 53 & 88 & 53 & 89 & 53 \\
\hline 0.3 & 86 & 60 & 87 & 59 & 90 & 59 \\
\hline 0.4 & 82 & 64 & 83 & 63 & 87 & 63 \\
\hline 0.5 & 74 & 65 & 76 & 65 & 80 & 64 \\
\hline 0.6 & 65 & 67 & 69 & 66 & 73 & 65 \\
\hline 0.7 & 52 & 69 & 57 & 67 & 63 & 66 \\
\hline 0.8 & 36 & 70 & 41 & 68 & 49 & 67 \\
\hline
\end{tabular}




\section{CONCLUDING REMARKS}

The paper presents a novel method to address the problem of AMMPD. The novelty originates from the tackling of the challenging issues of detecting and locating plagiarised subsegments between two suspicious music melodies through an image-based approach in spatial domain. The proposed method performs mainly the path exploration over a 2-D binary mask. The key contribution of the proposed method is a feasible solution to the two major issues in AMMPD, including (1) the inexact matching of noisy and inaccurate pitches of music audio and (2) the fast detection and positioning of similar subsegments between suspicious music audio. In fact, the proposed method is applicable to not only the applications of AMMPD, but also the applications of querying by humming/singing (QBSH).

\section{ACKNOWLEDGEMENTS}

This paper is supported by the Ministry of Science and Technology, Taiwan, under the project with the grant numbers 105-2221-E-259 -029 -.

\section{REFERENCES}

[1] Chung-Che Wang, Jyh-Shing Roger Jang, and Wennen Wang, "An improved query by singing/humming systemusing melody and lyrics information.," in ISMIR. Citeseer, 2010, pp. 45-50.

[2] Hung-Ming Yu, Wei-Ho Tsai, and Hsin-Min Wang, "A query-by-singing system for retrieving karaoke music,”IEEE Transactions on multimedia, vol. 10, no. 8, pp. 1626-1637, 2008.

[3] Yunjing Wang, "Similarity matching method for music melody retrieval," Journal of Multimedia, vol. 8, no. 4, pp.386-393, 2013.

[4] Donald J Berndt and James Clifford, "Using dynamic time warping to find patterns in time series." in KDDworkshop. Seattle, WA, 1994, vol. 10, pp. 359-370.

[5] Vassilis Athitsos, Panagiotis Papapetrou, Michalis Potamias, George Kollios, and Dimitrios Gunopulos, "Approximate embedding-based subsequence matching of time series," in Proceedings of the 2008 ACM SIGMOD International Conference on Management of data. ACM, 2008, pp. 365378.

[6] Mike Paterson and Vlado Dan` c' 1k, "Longest common subsequences," in International Symposium on MathematicalFoundations of Computer Science. Springer, 1994, pp. 127-142.

[7] Hwei-Jen Lin, Hung-Hsuan Wu, and Chun-Wei Wang, "Music matching based on rough longest common subsequence.,” J. Inf. Sci. Eng., vol. 27, no. 1, pp. 95-110, 2011.

[8] Li Hui, Bei-qian Dai, and Lu Wei, “A pitch detection algorithm based on amdf and acf," in 2006 IEEE InternationalConference on Acoustics Speech and Signal Processing Proceedings. IEEE, 2006, vol. 1, pp. I-I.

[9] Roger Chang, "MIR-QBSH dataset," http://mirlab.org/dataSet/public/. 


\section{AUTHORS}

Cheng-Chin Chiang received his Ph.D. degree in the Department of Computer Science and Information Engineering from National Chiao Tung University in Taiwan in 1993. He is now a professor at the Department of Computer Science and Information Engineering in National Dong Hwa University. His research interests include neural networks, pattern recognition, machine learning, multimedia processing and analysis, and virtual reality and augmented reality.

Mu-Syuan Sie received his Master degree in the Department of Computer Science and Information Engineering from National Dong Hwa University in Taiwan in 2015. He is now a multimedia system software engineer in MediaTek Inc. His research interests include human-machine interactions, machine learning, pattern recognition, and multimedia retrieval.

Hsiu-Chun Yangis pursuing his Master degree in the Department of Computer Science and Information Engineering from National Dong Hwa University. His research interests include machine learning, pattern recognition, and musical informationprocessing and retrieval.

Yi-Le Liuis pursuing his Master degree in the Department of Computer Science and Information Engineering from National Dong Hwa University. His research interests include machine learning, neural networks, pattern recognition, and content-based image retrieval.
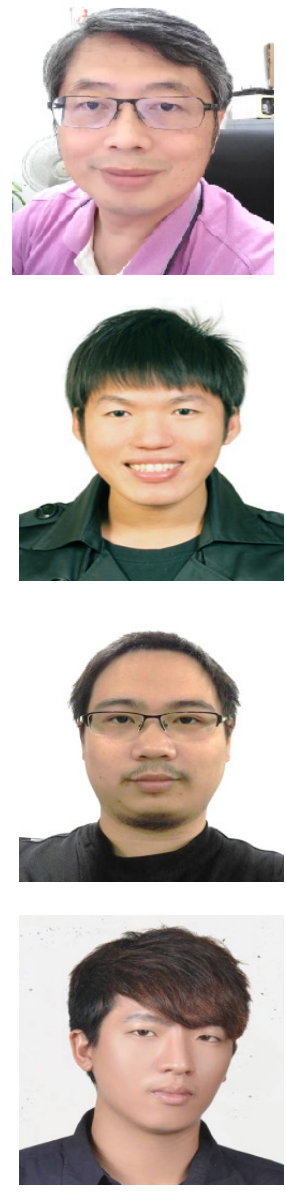